\title{
AUTOMATED SEARCH FOR LSB GALAXIES IN THE NEARBY UNIVERSE
}

\author{
Z. MORSHIDI, J.I. DAVIES AND R.M. SMITH \\ Dept. of Physics and Astronomy, University of Wales, Cardiff \\ P.O.Box 913, Cardiff CF2 3YB, U.K.
}

\section{Introduction}

We have carried out a large area survey for nearby LSB galaxies to try and answer the following questions:

1. How are LSB galaxies distributed relative to the normal HSB galaxies? Do they have the same clustering properties suggesting that they trace the same mass distribution?

2. What are the implications of the clustering properties on galaxy formation scenarios?

3. Can they account for faint blue galaxies at higher redshifts?

4. Is there any difference between the field and cluster LSB galaxies?

\section{The Survey}

We have devised an automated technique to pick out local LSB galaxies using 65 UKSTU photographic plates corresponding to $2187 \mathrm{sq}^{\circ}$. We have imposed stringent selection criteria in order to minimise the background contamination. Our galaxies have $\mu_{0} \geq 22.5 \mathrm{~B}$ mag arcsec ${ }^{-2}, \alpha \geq 3.0$ arcseconds and $B_{T} \leq 20.0$. Our survey areas are confined to the Fornax Cluster, the NGC1400, Sculptor and Dorado Groups and the fields between them. The survey, which was carried out in three stages over a period of 2 years, has produced detections of 2435 LSB galaxies (32 galaxies per field). We have estimated the background contamination in three ways: first, by a limited redshift sample, secondly, by Ferguson's (1989) classification on the Fornax plate and finally, by a numerical simulation of background galaxies. The first method produced an estimate of 14 galaxies per field, while the second and the third methods produced the same estimate of 19 galaxies.

\section{Results}

Figure 1 shows the spatial distributions of the dwarf galaxies. 19 galaxies representing the background contaminants have been randomly removed from each field. The data for the bright galaxies were taken from the RC3 (de Vaucouleur 1991). The results of the dwarf simulations indicate that, if the local Luminosity Function of Driver (1994) is correct, we should have detected 54 dwarf galaxies in each field if the intrinsic surface brightness follows the $\mu_{0}-M_{B}$ relation and 68 galaxies if they have a flat intrinsic surface brightness distribution. Yet our data indicate an average detection rate of 13 nearby galaxies per field. On average, we have detected less galaxies than that predicted by the Driver model. Either Driver's Luminosity Function is incorrect or the dwarf galaxies in the field have faded below our detection threshold and the field galaxies have different surface brightness distributions than those in the clusters. Babul \& Rees (1992) suggest that the dwarf ellipticals in the clusters can maintain their luminosities while those in the field are more susceptible to gas loss thus ceasing their star formation activities and, consequently, fading more quickly than the cluster dwarfs. 


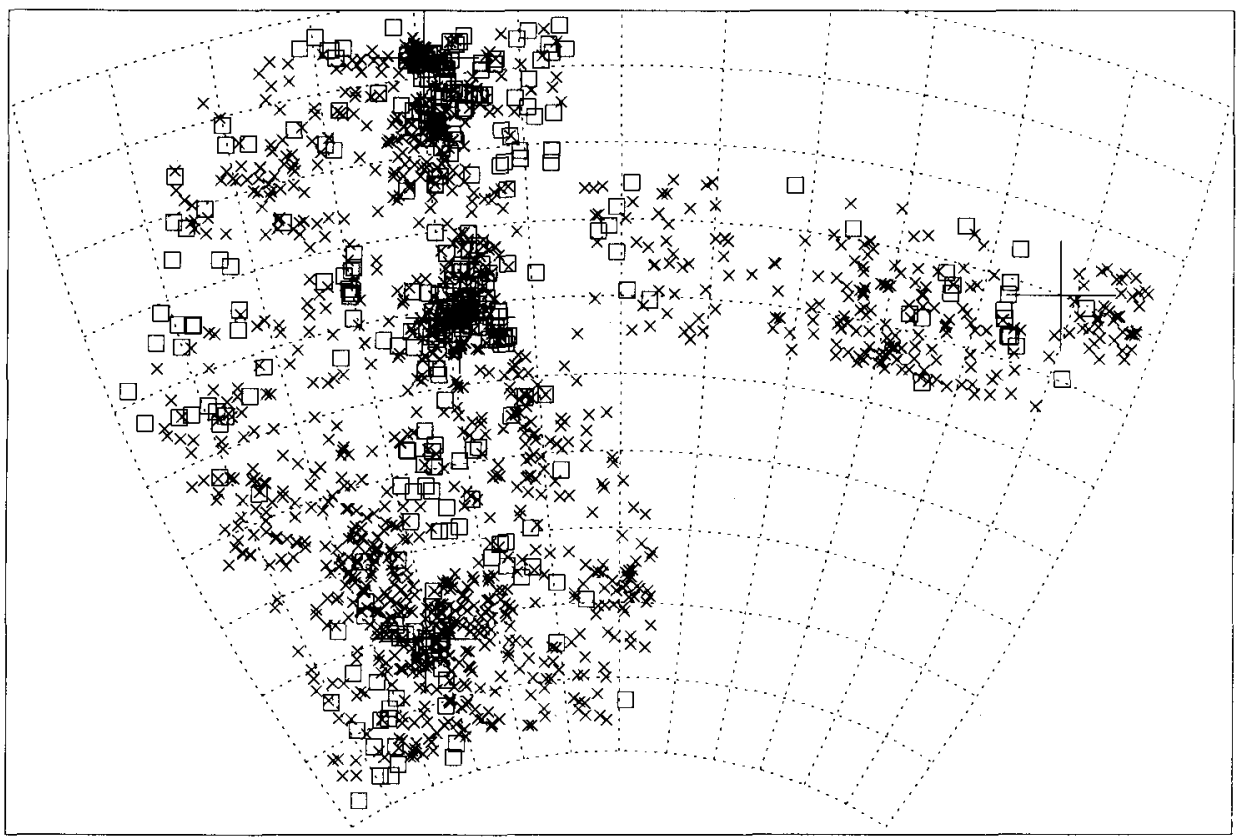

Figure 1. The distribution of nearby LSB galaxies $(x)$ and the bright galaxies ( $\square$ ) in an equal-area plot. The large + are the centres of the cluster and groups.

\section{References}

Babul, A. and Rees, M.J. (1992) MNRAS, 255, 346

de Vaucouleur, G.H. (1991) Third Ref. Cat. of Bright Galaxies, Springer-Verlag, New York

Driver, S.P. (1994) PhD Thesis, University of Wales Cardiff

Ferguson, H.C. (1989) $A J, 98,367$

Freeman, K.C. (1970) ApJ, 160, 811

Loveday, J., Peterson, B.A.,Efstathiou, G. and Maddox, S.J. (1992) ApJ, 390, 338 